This item was submitted to Loughborough's Research Repository by the author.

Items in Figshare are protected by copyright, with all rights reserved, unless otherwise indicated.

\title{
Decentralised standoff tracking of moving targets using adaptive sliding mode control for UAVs
}

\section{PLEASE CITE THE PUBLISHED VERSION}

http://dx.doi.org/10.1007/s10846-013-9864-0

\section{PUBLISHER}

(c) Springer Science and Business Media

\section{VERSION}

AM (Accepted Manuscript)

\section{PUBLISHER STATEMENT}

This work is made available according to the conditions of the Creative Commons Attribution-NonCommercialNoDerivatives 4.0 International (CC BY-NC-ND 4.0) licence. Full details of this licence are available at: https://creativecommons.org/licenses/by-nc-nd/4.0/

\section{LICENCE}

CC BY-NC-ND 4.0

\section{REPOSITORY RECORD}

Oh, Hyondong, Seungkeun Kim, Antonios Tsourdos, and Brian A. White. 2014. "Decentralised Standoff Tracking of Moving Targets Using Adaptive Sliding Mode Control for Uavs". figshare. https://hdl.handle.net/2134/16087. 


\title{
Decentralised Standoff Tracking of Moving Targets Using Adaptive Sliding Mode Control for UAVs
}

\author{
Hyondong Oh, Seungkeun Kim*, Antonios Tsourdos, and Brian A. White
}

\begin{abstract}
This paper proposes a decentralised vector field guidance algorithm for coordinated standoff tracking of a ground moving target by multiple UAVs. In particular, this study introduces additional adaptive terms in an existing sliding mode control concept for standoff tracking guidance, in order to reduce the effect of unmodelled dynamics and disturbances. Decentralised angular separation control between UAVs, in conjunction with decentralised estimation, is also introduced using either velocity or orbit radius change by different information/communication structures. Numerical simulations are performed to verify the feasibility and benefits of the proposed approach under a realistic ground vehicle tracking scenario, using multiple UAVs having unknown parameters in the heading-hold autopilot.

Index Terms-UAV, Standoff tracking, Vector field guidance, Sliding mode control, Decentralised estimation and guidance
\end{abstract}

\section{INTRODUCTION}

In recent decades, the benefits of using cooperative unmanned aerial vehicles (UAVs) have been actively investigated for both military and civil applications. The intelligent and autonomous cooperation of multiple small UAVs operating in a team/swarm offers revolutionary capabilities: improved situation awareness, higher mission success rate, significant reductions in manpower and risk to humans, the ability to perform in hostile and hazardous environments. Especially, airborne surveillance and subsequent tracking of a ground moving target have become one of the important

Hyondong Oh is with the Department of Computing, University of Surrey, Guildford, Surrey, GU2 7XH, United Kingdom, e-mail: h.oh@surrey.ac.uk

* Seungkeun Kim (Corresponding author) is with the Department of Aerospace Engineering, Chungnam National University, 99 Daehak-ro, Yuseong-gu, Daejeon 305-764, Republic of Korea, e-mail: skim78@cnu.ac.kr.

Antonios Tsourdos and Brian A. White are with the Department of Engineering Physics, Cranfield University, Cranfield, MK43 0AL, United Kingdom, e-mail: \{a.tsourdos, b.a.white\}@cranfield.ac.uk capabilities of UAVs, required to increase overall knowledge to surrounding environment and to take proactive measures.

In performing such missions, once a target of interest is detected by reconnaissance or surveillance systems, further monitoring might be required to obtain closer and higher-resolution surveillance data, by approaching more closely and tracking it persistently [1]. This is not an easy task due to unknown or even adversarial/evasive target manoeuvres, as well as kinematic constraints of UAVs. For this, a standoff line-of-sight (LOS) tracking concept is introduced, which keeps a certain relative distance (termed standoff distance) from a moving target, resulting in a circular loitering pattern, in order to track without being noticed and to keep it within the field-of-view (FOV) of a sensor. Coordinated standoff tracking using multiple UAVs is also proposed by distributing UAVs with a prescribed inter-vehicle angular separation on the same standoff orbit. It can provide the better estimation accuracy for target information [2] as well as more robust tracking performance in case of sensing failure or LOS block to the target by obstacles [3].

Lawrence [4] first proposed the application of Lyapunov vector fields for standoff coordination of multiple UAVs, which was further investigated by Frew et al. [5] to include phase keeping as well as standoff tracking. They invented a decoupled control structure in which speed and heading rate are separately controlled for standoff distance and phase-angle keeping, respectively. Summers et al. [6] extended this phase keeping idea with a variable airspeed controller to multiple UAVs using information architectures in vehicle formations. Unlike decoupled control structures, Kim et al. [7] proposed a nonlinear model predictive approach to find the heading and velocity control inputs si- 
multaneously using a coupled structure, providing optimal performance in terms of tracking as well as control efforts. Oh et al. [8] used the solution of differential geometry between the UAV and a target, which brings several advantages along with its inherent simplicity: rigorous stability, explicit use of a target velocity, and tuning parameter reduction. Oh et al. [9] also introduced cooperative standoff tracking of groups of multiple targets using Lyapunov vector fields and an online local replanning strategy.

Note that, considering small allowable velocity bounds of the UAV and time delay in the velocity control, the convergence to the variable velocity command might be slow or hard to achieve; thus, maintaining UAVs at their nominal and fuel efficient speed is desirable in terms of tracking performance, as well as from a mission duration point of view. In this regard, Kingston et al. [10] introduced a sliding mode control concept for circular formation and orbit radius change without velocity control for phase keeping of multiple UAVs. Sepulchre et al. [11] and Paley et al. [12] applied the collective control of multi-agent system to stabilise symmetric circular formation around the target using unit speed vehicles. Note also that policies that maintain the optimal standoff distance while allowing the angular separation to vary can be better in terms of estimation accuracy than those that maintain optimal angular separation with orbit radius change while compromising the standoff distance [2]. Therefore, a tracking guidance algorithm that explicitly exploits both orbit radius and velocity control concept is required to be developed within a unified framework.

This paper proposes coordinated standoff tracking guidance algorithms of a ground moving target using sliding model control (SMC), based on vector field approach. Motivated by the aforementioned work [10], this study first exploits additional adaptive terms in the existing vector field approach using sliding mode control, in order to reduce the effect of unmodelled dynamics and disturbances in the heading-hold autopilot. Then, decentralised angular separation control for multiple UAVs is introduced using either velocity or orbit radius change with a constant airspeed by different information structures, following [6], [10]. Note that, it is hard to have a centralised system that handles entire information and controls for a team of UAVs considering communication and computation constraints; thus, control laws are decentralised using local information about a target and neighbouring UAVs. Moreover, in order to obtain accurate position and motion information of a moving target to be used for UAV guidance, as well as to cope with the proposed decentralised guidance approach, this study applies the decentralised extended Information filter (DEIF). Numerical simulations are extensively performed to verify the feasibility and compare benefits of the proposed guidance algorithms, under a realistic ground vehicle tracking scenario. In the simulations, it is assumed that multiple UAVs have unknown parameters in the system.

The remainder of this paper is organised as follows. Several vector field approaches are first reviewed in Section II, and the tangent vector field guidance (TVFG) using the adaptive SMC follows in Section III. Section IV introduces the decentralised TVFG for angular separation between UAVs. Section V explains a decentralised target localisation methodology. Section VI presents numerical simulation results to verify the performance and the properties of the proposed algorithm. Lastly, conclusions and future work are given in Section VII.

\section{Review on Vector Fields Strategies}

This section briefly reviews several recent vector field guidance approaches for standoff tracking of a ground moving target, including their formulation and numerical example results for stationary target tracking.

\section{A. System dynamics}

Assuming each UAV has a low-level flight controller such as SAS (Stability Augmentation System) and CAS (Controllability Augmentation System) for heading and velocity hold functions, this study aims to design guidance inputs to this low-level controller for standoff target tracking. Consider a two-dimensional UAV kinematic model 
[7] as:

$$
\left(\begin{array}{c}
\dot{x} \\
\dot{y} \\
\dot{\psi} \\
\dot{v} \\
\dot{\omega}
\end{array}\right)=f(\mathbf{x}, \mathbf{u})=\left(\begin{array}{c}
v \cos \psi \\
v \sin \psi \\
\omega \\
-\frac{1}{\tau_{v}} v+\frac{1}{\tau_{\dot{P}}} u_{v} \\
-\frac{1}{\tau_{\omega}} \omega+\frac{1}{\tau_{\omega}} u_{\omega}
\end{array}\right)
$$

where $\mathbf{x}=(x, y, \psi, v, \omega)^{T}$ are the inertial position, heading, speed and yaw rate of the UAV, respectively. $\tau_{v}$ and $\tau_{\omega}$ are time constants for considering actuator delay. $\mathbf{u}=\left(u_{v}, u_{\omega}\right)^{T}$ are the commanded speed and turning rate constrained by the following dynamic limits of a fixed-wing UAV:

$$
\begin{aligned}
\left|u_{v}-v_{0}\right| & \leq v_{\max } \\
\left|u_{\omega}\right| & \leq \omega_{\max }
\end{aligned}
$$

where $v_{0}$ is a nominal speed of UAV. The continuous UAV model in Eq. (1) can be discretised by Euler integration into:

$$
\mathbf{x}_{k+1}=f_{d}\left(\mathbf{x}_{k}, \mathbf{u}_{k}\right)=\mathbf{x}_{k}+T_{s} f\left(\mathbf{x}_{k}, \mathbf{u}_{k}\right)
$$

where $\mathbf{x}_{k}=\left(x_{k}, y_{k}, \psi_{k}, v_{k}, \omega_{k}\right)^{T}, \quad \mathbf{u}_{k}=$ $\left(u_{v k}, u_{\omega k}\right)^{T}$, and $T_{s}$ is a sampling time. If the frequencies of the tracking guidance and autopilot are not too close, it is common to initially design and verify the guidance law and control algorithm separately. Therefore, like in many literatures considering similar guidance problems [5], [7], [10], above simple kinematics is used for the UAV model. However, the final validation needs to be made with higher complexity simulation models and flight tests, and these remain as future work.

\section{B. Lyapunov vector field}

Let us consider the Lyapunov vector field first, which was initially proposed by Lawrence [4] and further developed by Frew et al. [5] as:

$$
V(x, y)=\left(r^{2}-r_{d}^{2}\right)^{2}
$$

where $r=\sqrt{\delta x^{2}+\delta y^{2}}=\sqrt{\left(x-x_{t}\right)^{2}+\left(y-y_{t}\right)^{2}}$ is the distance of the UAV from the ground vehicle. Herein $\left(x_{t}, y_{t}\right)$ is the position of the ground vehicle which can be estimated from the tracking filter, and $r_{d}$ is a desired standoff distance from the UAV to the ground vehicle. Differentiating Eq. (5) gives:

$$
\dot{V}(x, y)=\nabla V[\dot{x}, \dot{y}]^{T} .
$$

The Lyapunov vector field uses the following desired velocity $\left[\dot{x}_{d}, \dot{y}_{d}\right]^{T}$ :

$\left[\begin{array}{c}\dot{x}_{d} \\ \dot{y}_{d}\end{array}\right]=\frac{-V_{d}}{k_{l} r\left(r^{2}+r_{d}^{2}\right)}\left[\begin{array}{l}\delta x\left(r^{2}-r_{d}^{2}\right)+\delta y\left(2 r r_{d}\right) \\ \delta y\left(r^{2}-r_{d}^{2}\right)-\delta x\left(2 r r_{d}\right)\end{array}\right]$

where $k_{l}$ is positive constant, and $V_{d}$ is a desired UAV speed. Note that $k_{l}$, newly introduced in this study, can be used to adjust the converging speed of the generated field to the standoff circle. The desired heading can be decided using the desired two dimensional velocity components in Eq. (7) as:

$$
\psi_{d}=\tan ^{-1} \frac{\dot{y}_{d}}{\dot{x}_{d}} .
$$

The guidance command $u_{\omega}$ for turn rate is selected as the sum of proportional feedback and feedforward terms as:

$$
u_{\omega}=-k_{\psi}\left(\psi-\psi_{d}\right)+\dot{\psi}_{d}
$$

where $\dot{\psi}_{d}=4 v_{d} \frac{r_{d} r^{2}}{\left(r^{2}+r_{d}^{2}\right)^{2}}$ can be obtained by differentiating Eq. (8).

\section{Supercritical Hopf bifurcation}

The second vector field is the supercritical Hopf bifurcation which was initially proposed in [13] based on the theory of [14]. The Supercritical Hopf bifurcation is known to mathematically produce the spiral trajectory which converges to a limit cycle; no matter where the starting position is located. This property is similar to that of the Lyapunov vector field. Let us consider the following desired vector field based on the system equation of the Supercritical Hopf bifurcation:

$$
\left[\begin{array}{c}
\dot{x}_{d} \\
\dot{y}_{d}
\end{array}\right]=\left[\begin{array}{c}
\frac{\delta x}{k_{s} r_{d}^{2}}\left(r_{d}^{2}-\delta x^{2}-\delta y^{2}\right)-\delta y \\
\frac{\delta y}{k_{s} r_{d}^{2}}\left(r_{d}^{2}-\delta x^{2}-\delta y^{2}+\delta x\right.
\end{array}\right]
$$

where $k_{s}$ is a positive constant, and the definitions of the other variables are the same as those of the Lyapunov vector field.

\section{Tangent vector field}

The tangent vector field is generated by a desired heading angle as shown in Fig. 1, given by [10]:

$$
\psi_{d}=\psi_{p}+\tan ^{-1}\left(k_{t} d\right)
$$

where $d=r-r_{d}$ is the distance of the UAV from the desired standoff orbit, and $\psi_{p}$ is tangent to the 


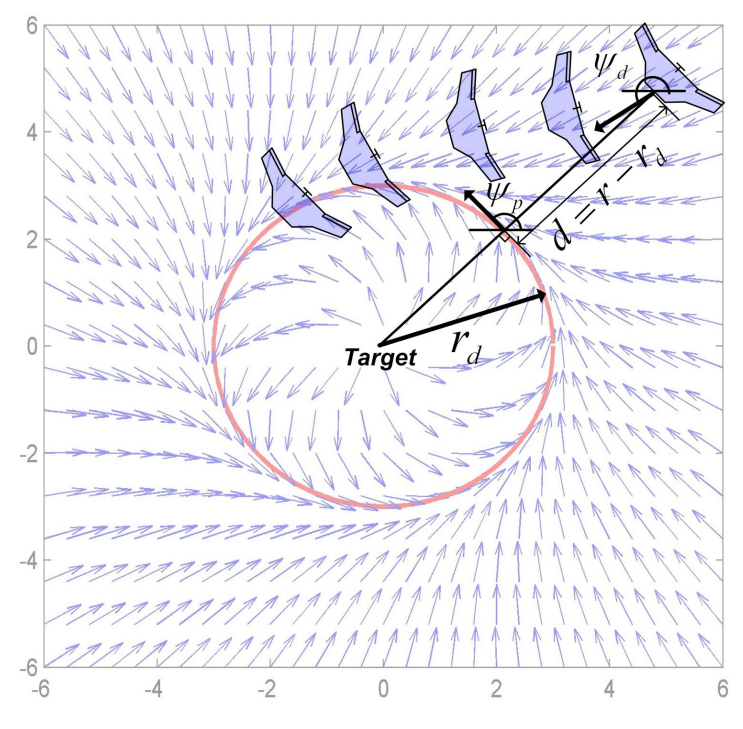

Fig. 1. Geometry of tangent vector field

standoff orbit along the ray connecting the UAV and the target position as:

$$
\psi_{p}=\theta+\frac{\pi}{2}
$$

where $\theta=\tan ^{-1}(\delta y / \delta x)$. The convergence analysis of a desired heading angle to $\psi_{p}$ can be found in [10].

Figure 2 shows the example of UAV tracking trajectories for a stationary target using different vector field approaches. Although aforementioned three vector fields have different characteristics, they can generate a similar trajectory as red dashed lines in Fig. 2, by adjusting the field gain such as $k_{l}, k_{s}$ and $k_{t}$. It is worth noting that the field gain should be determined not to exceed the turning rate constraint of the vehicle, $\omega_{\max }$, along with a control gain. This study uses a tangent vector field approach since it allows to easily apply a sliding mode control concept, as well as the orbit radius change for angular separation control between UAVs.

\section{TAngent Vector Field Guidance WITH ADAPTIVE SMC}

\section{A. Adaptive sliding mode control}

This section presents the tangent vector field guidance (TVFG) strategy using a sliding mode control concept. First, an UAV is assumed to follow a first-order heading dynamics by the autopilot, as given:

$$
\dot{\psi}=\alpha\left(\psi_{c}-\psi\right)+\nu
$$

where $\psi_{c}$ is the commanded heading, $\alpha$ is a known positive constant that characterises the speed of response of heading-hold autopilot loop, and $\nu$ represents unmodelled dynamics or disturbances of the autopilot loop. Let us first consider a conventional proportional-derivative (PD) type controller. If $\alpha$ were accurately known and $\nu$ were small enough to ignore, the guidance command $\psi_{c}$, to obtain the desired heading $\psi_{d}$ in Eq. (11), would be selected as the sum of proportional feedback and feedforward term as:

$$
\psi_{c}=\psi+\frac{1}{\alpha}\left(\dot{\psi}_{d}-k_{\psi}\left(\psi-\psi_{d}\right)\right)
$$

where $k_{\psi}$ represents a proportional gain.

However, in general, $\alpha$ is difficult to determine experimentally and inevitably contains error in its estimated value, and $\nu$ can have a considerable value with known bounds due to unmodeled dynamics, disturbances or faults. Since these factors could result in less precise target tracking, this study uses adaptive sliding mode control, which estimates and compensates the effect of unknown parameters.

First of all, let us define a sliding surface as:

$$
S=e+k_{I} \int_{0}^{t} e d \tau
$$

where $e=\psi-\psi_{d}$ is a tracking error, and $k_{I}$ is an integral gain. Differentiating the sliding surface with respect to time gives:

$$
\dot{S}=\dot{\psi}-\dot{\psi}_{d}+k_{I} e=\alpha\left(\psi_{c}-\psi\right)+\nu-\dot{\psi}_{d}+k_{I} e .
$$

Consider a Lyapunov function candidate: $W_{1}=$ $\frac{1}{2} S^{2}$, and take the derivative to obtain:

$$
\dot{W}_{1}=S \dot{S}=S\left(\alpha\left(\psi_{c}-\psi\right)+\nu-\dot{\psi}_{d}+k_{I} e\right)
$$

Then, the control command can be selected as:

$$
\psi_{c}=\psi+\frac{1}{\hat{\alpha}}\left(-\hat{\nu}+\dot{\psi}_{d}-k_{I} e-c_{1} S-c_{2} \operatorname{sign}(S)\right)
$$

where $\dot{\psi}_{d}$ is a derivative of Eq. (11), $\hat{\alpha}$ and $\hat{\nu}$ are the estimation of $\alpha$ and $\nu$, respectively, $c_{1}$ and $c_{2}$ 


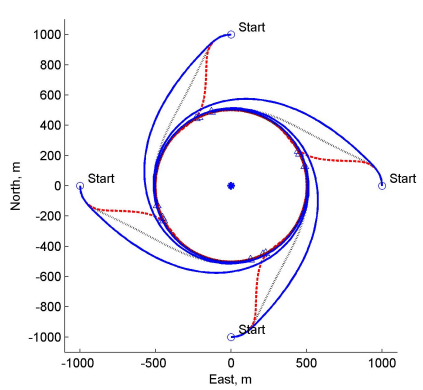

(a) Lyapunov vector field

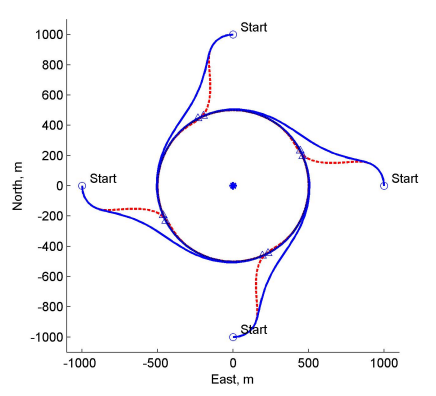

(b) Supercritical Hopf bifurcation field

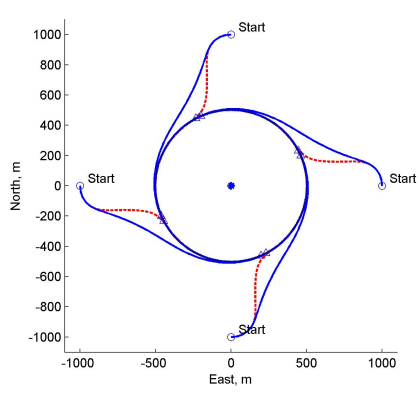

(c) Tangent vector field

Fig. 2. Example UAV trajectories for a stationary ground target using vector field approaches

are a positive constant, and

$$
\operatorname{sign}(x)= \begin{cases}1, & \text { if } x>0 \\ 0, & \text { if } x=0 \\ -1, & \text { if } x<0\end{cases}
$$

Rearrange Eq. (17) using $\psi_{s}=\psi_{c}-\psi$ as:

$$
\begin{aligned}
\dot{W}_{1} & =S\left(\alpha \psi_{s}+\nu-\dot{\psi}_{d}+k_{I} e\right) \\
& =S\left\{(\alpha-\hat{\alpha}) \psi_{s}+\hat{\alpha} \psi_{s}+\nu-\dot{\psi}_{d}+k_{I} e\right\} \\
& =S\left\{\tilde{\alpha} \psi_{s}+\hat{\alpha}\left(\psi_{c}-\psi\right)+\nu-\dot{\psi}_{d}+k_{I} e\right\}
\end{aligned}
$$

where $\tilde{\alpha}=\alpha-\hat{\alpha}$. Substituting $\psi_{c}$ in Eq. (18) into Eq. (20) yields:

$$
\begin{aligned}
\dot{W}_{1}= & S\left\{\tilde{\alpha} \psi_{s}-\hat{\nu}+\dot{\psi}_{d}-k_{I} e-c_{1} S-c_{2} \operatorname{sign}(S)\right. \\
& \left.+\nu-\dot{\psi}_{d}+k_{I} e\right\} \\
= & S\left\{\tilde{\nu}-c_{1} S-c_{2} \operatorname{sign}(S)+\tilde{\alpha} \psi_{s}\right\}
\end{aligned}
$$

where $\tilde{\nu}=\nu-\hat{\nu}$.

To obtain the adaptation rule for the parameter estimations of $\hat{\nu}$ and $\hat{\alpha}$, consider another Lyapunov function candidate $W_{2}$ as:

$$
W_{2}=\frac{1}{2} S^{2}+\frac{1}{2} \gamma_{\nu}^{-1} \tilde{\nu}^{2}+\frac{1}{2} \gamma_{\alpha}^{-1} \tilde{\alpha}^{2}
$$

where $\gamma_{\nu}$ and $\gamma_{\alpha}$ are positive constants. Differentiating Eq. (22) and using Eq. (21) gives:

$$
\begin{aligned}
\dot{W}_{2}= & S\left(\tilde{\nu}-c_{1} S-c_{2} \operatorname{sign}(S)+\tilde{\alpha} \psi_{s}\right) \\
& +\gamma_{\nu}^{-1} \tilde{\nu} \dot{\tilde{\nu}}+\gamma_{\alpha}^{-1} \tilde{\alpha} \dot{\tilde{\alpha}}=-c_{1} S^{2}-c_{2}|S| \\
& +\tilde{\nu}\left(S-\gamma_{\nu}^{-1} \dot{\hat{\nu}}\right)+\tilde{\alpha}\left(\psi_{s} S-\gamma_{\alpha}^{-1} \dot{\hat{\alpha}}\right)
\end{aligned}
$$

where the relation $\dot{\tilde{\nu}}=-\dot{\hat{\nu}}$ and $\dot{\tilde{\alpha}}=-\dot{\hat{\alpha}}$ are used under the assumption that $\nu$ and $\alpha$ are constant.
Then, the adaptation law for $\hat{\nu}$ and $\hat{\alpha}$ can be obtained as:

$$
\begin{aligned}
\dot{\hat{\nu}} & =\gamma_{\nu} S \\
\dot{\hat{\alpha}} & =\gamma_{\alpha} \psi_{s} S .
\end{aligned}
$$

Then,

$$
\dot{W}_{2}=-c_{1} S^{2}-c_{2}|S| \leq 0
$$

from which it can be concluded that $S$ goes to zero in finite time, and finally the error state $e$ tends to zero by LaSalle-Yoshizawa theorem [15]. This means that the heading angle $\psi$ of the UAV can follow the desired heading $\psi_{d}$ provided from Eq. (11) in spite of model uncertainties in $\alpha$ and $\nu$. To avoid the chattering problem which results from the discontinuity of sign function, this study replaces the sign function in the control command with the continuous saturation function as given:

$$
\text { sat }\left(\frac{S}{\epsilon}\right)= \begin{cases}\frac{S}{\epsilon}, & \text { if }\left|\frac{S}{\epsilon}\right| \leq 1 \\ \operatorname{sign}\left(\frac{S}{\epsilon}\right), & \text { otherwise }\end{cases}
$$

where $\epsilon>0$ represents the width of the boundary layer around the sliding surface.

\section{B. Taking a target velocity into account}

If the velocity of a ground moving target can be estimated (as will be described in the next section), the vector field can be adjusted in order to improve tracking guidance performance, by taking a target velocity into account. Let us consider the behaviour of a point orbiting a constant speed target at a fixed radius $r_{d}$, then, the position of the point can be expressed as:

$$
\begin{aligned}
& x_{t p}=r_{d} \cos \theta+T_{x} t \\
& y_{t p}=r_{d} \sin \theta+T_{y} t
\end{aligned}
$$


where $T_{x}$ and $T_{y}$ are the speed of the target in $x$ and $y$ axis, respectively. Then, the path heading angle for a moving target can be obtained as:

$$
\psi_{p}=\tan ^{-1}\left(\frac{\dot{y}_{t p}}{\dot{x}_{t p}}\right) .
$$

Maintaining the desired UAV speed $V_{d}$ leads the following condition to be met:

$$
\begin{aligned}
V_{g}^{2} & =\left(\dot{x}_{t p}\right)^{2}+\left(\dot{y}_{t p}\right)^{2} \\
& =\left(-r_{d} \dot{\theta} \sin \theta+T_{x}\right)^{2}+\left(r_{d} \dot{\theta} \cos \theta+T_{y}\right)^{2}
\end{aligned}
$$

By solving above equation with respect to $\dot{\theta}$, the final path heading angle $\psi_{p}$ and the modified $\psi_{d}$ can be obtained. Note that there will be an error due to the estimations of $T_{x}$ and $T_{y}$ which eventually could make the closed-loop dynamics unstable through the feedforward path. This can be addressed by acquiring as accurate estimations as possible (which will be discussed in Section V) and selecting conservative $c_{1}$ and $c_{2}$ constants in the SMC controller in consideration of a bounded error for $\psi_{d}$.

\section{Decentralised TVFG FOR ANgular SEPARATION}

In performing a coordinated target tracking mission, UAVs should keep a prescribed inter-vehicle angular separation to maximise sensor coverage or enhance the estimation accuracy of the target information, while maintaining a standoff distance from a target. To do so, this section introduces decentralised angular separation control of multiple UAVs using either velocity or orbit radius change by different information architectures. It builds upon a rigid graph theory utilising: asymmetric minimally persistent leader-follower and symmetric nonminimally persistent based on the previous study [6].

\section{A. Minimally persistent leader-follower informa- tion architecture}

In this architecture, one of UAVs (leader) follows the standoff orbit around a target with desired airspeed and orbit radius using the TVFG with the adaptive SMC. The remaining vehicles (followers) maintain the same orbit; however, they keep a prescribed angular spacing with the neighboring vehicle ahead of it by adjustments of airspeed or orbit radius. This architecture can be modelled by a directed graph as illustrated in Fig. 3(a), and requires a minimum possible number of communication/sensing links to achieve the circular orbit and angular spacing.

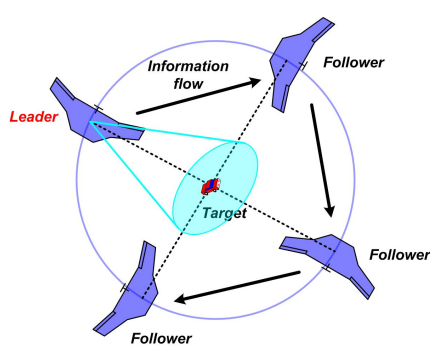

(a) Minimally persistent

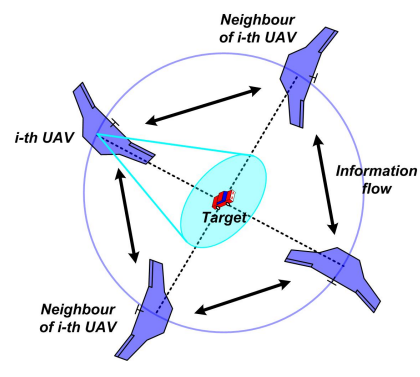

(b) Nonminimally persistent
Fig. 3. Illustration of information architectures

1) Orbit radius change: Let $n$-th UAV have the constant desired orbit radius $r_{d}$, and the remaining $n-1$ UAVs have a variable orbit radius by changing the desired heading angle of the tangent vector field as:

$$
\psi_{i}^{d}=\psi_{i}^{p}+\tan ^{-1}\left(k_{t}\left(d_{i}-k_{o}^{m} \delta \theta_{i}^{m}\right)\right)
$$

where $k_{o}^{m}$ is a control gain weighting the convergence to a desired orbit, and $\delta \theta_{i}^{m}$ is $i$-th angular spacing error given by:

$$
\delta \theta_{i}^{m}=\theta_{i+1}-\theta_{i}-\theta_{d}
$$

where $\theta_{d}$ is the desired angular separation between UAVs. Note that, $d_{i}=r_{i}-r_{d}$ is a distance from the desired orbit to the UAV position in the normal TVFG as Eq. (11); now it is modified by additional term $\left(k_{o}^{m} \delta \theta_{i}^{m}\right)$ according to the angular spacing error, resulting in the temporary change of orbit radius, as illustrated in Fig. 4. In this figure, while the leader UAV is staying on the desired orbit, the follower UAV goes to the orbit of larger radius to decrease the angular separation error with the amount of $\delta \theta_{i}$ to the leader; the rest of followers will respond accordingly. The difference of time spent on the different orbits allows for the control of the angular separation of UAVs.

2) Velocity change: Similarly to the orbit radius change case, let $n$-th UAV have the desired velocity input $V_{d}$, and the remaining $n-1 \mathrm{UAV}$ s have 


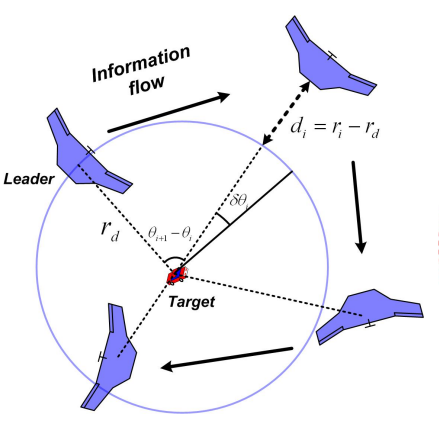

(a) Original orbit radius

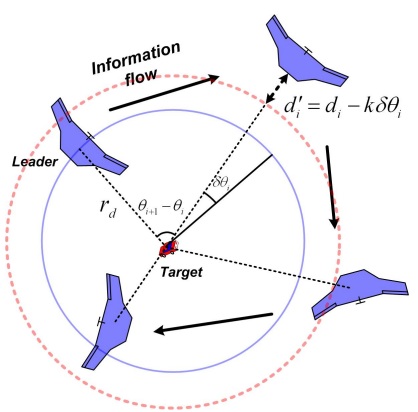

(b) Modified orbit radius
Fig. 4. Illustration of orbit radius change in minimally persistent case

a variable velocity input according to the angular spacing error as:

$$
u_{v i}=V_{d}+\Delta V_{\max } \tanh \left(k_{v}^{m} \delta \theta_{i}^{m}\right)
$$

where $k_{v}^{m}$ is a control gain, and $\delta \theta_{i}^{m}$ is the same as Eq. (32), and $\Delta V_{\max }>0$ is a design parameter to be met for a speed variation constraint of the UAV. When the angular separation is different from the desired value, a velocity input of each UAV is adjusted accordingly without changing an orbit radius, as illustrated in Fig. 5

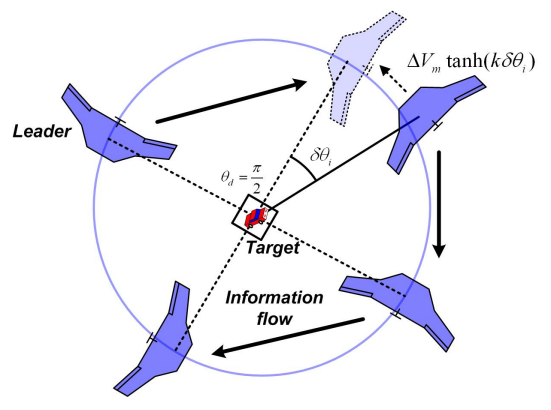

Fig. 5. Illustration of velocity change in minimally persistent case

\section{B. Nonminimally persistent information architec- ture}

In this architecture, the airspeed or orbit radius is adjusted such that each vehicle moves toward the midpoint of its two nearest neighbours on the standoff orbit. This is modelled by an undirected graph as illustrated in Fig. 3(b). This architecture does not need to know the number of engaging vehicles in advance, compared to the minimally persistent case that requires a desired separation angle $\theta_{d} \mathrm{~d}$; this control structure can be viewed as fully decentralised.
1) Orbit radius change: Let $i$-th UAV have a variable orbit radius by changing a heading command of the TVFG as:

$$
\psi_{i}^{d}=\psi_{i}^{p}+\tan ^{-1}\left(k_{t}\left(d_{i}+k_{o}^{n} \delta \theta_{i}^{n}\right)\right)
$$

where $k_{o}^{n}$ is a control gain, and $\delta \theta_{i}^{n}$ is $i$-th angular spacing error given by:

$$
\delta \theta_{i}^{n}=\theta_{i}-\frac{1}{2}\left(\theta_{i-1}+\theta_{i+1}\right)
$$

where a modulo $n$ cycle is formed around the circle as:

$$
\theta_{0}=\theta_{n}-2 \pi, \quad \theta_{n+1}=\theta_{1}+2 \pi
$$

2) Velocity change: Let the $n$ UAVs have a variable velocity input according to the angular spacing error as:

$$
u_{v i}=V_{d}-\Delta V_{\text {max }} \tanh \left(k_{v}^{n} \delta \theta_{i}^{n}\right)
$$

where $k_{v}^{n}$ is a control gain, and $\delta \theta_{i}^{n}$ is the same as Eq. (35).

\section{Decentralised Target Localisation}

The performance of the standoff tracking guidance algorithms proposed so far is strongly coupled with the sensing and estimation capability against the moving target on the ground. To produce appropriate tracking data, a GMTI (Ground Moving Target Indicator) is a well-suited radar sensor due to its wide-coverage, all-weather, day/night, and real-time capabilities [16]. From this sensor data such as range, azimuth, or elevation of the target with respect to the sensor location, a certain level of accurate estimation could be obtained using conventional filtering techniques. In order to further improve the estimation accuracy, as well as cope with the proposed decentralised guidance approach, this study applies a decentralised multisensor fusion algorithm.

Given that multiple UAVs carry out the process of tracking the same ground target, each UAV will obtain its own sensor measurement and executes the tracking filter separately. After each UAV receives the other's estimation via a communication link, it can run a decentralised data fusion. A ground target and sensor model are first formulated, information exchange between UAVs is briefly introduced, and then a decentralised extended Information filter (DEIF) algorithm [17], [18] follows. 


\section{A. Ground target and sensor model}

This study considers acceleration dynamics [19], [7] for a ground moving vehicle, which irregularly performs stop-and-go manoeuvres. UAVs are assumed to be equipped with a GMTI sensor to localise the position of target. Because the measurement of a GMTI is composed of range and azimuth of the target with respect to the radar location, the actual measurements is the relative range and azimuth with respect to the position of the UAV. The radar measurement $\mathbf{z}_{k}=\left(r_{k}, \phi_{k}\right)^{T}$ can be defined as the following nonlinear relation using the target position $\left(x_{k}^{t}, y_{k}^{t}\right)^{T}$ and the UAV position $\left(x_{k}, y_{k}\right)^{T}$ as:

$$
\begin{aligned}
\mathbf{z}_{k} & =\left(\begin{array}{l}
r_{k} \\
\phi_{k}
\end{array}\right)=h\left(\mathbf{x}_{k}^{t}\right)+\nu_{k} \\
& =\left(\begin{array}{c}
\sqrt{\left(x_{k}^{t}-x_{k}\right)^{2}+\left(y_{k}^{t}-y_{k}\right)^{2}} \\
\tan ^{-1} \frac{y_{k}^{t}-y_{k}}{x_{k}^{t}-x_{k}}
\end{array}\right)+\nu_{k}
\end{aligned}
$$

where $\nu_{k}$ is a measurement noise vector, and its noise covariance matrix is defined as:

$$
V\left[\nu_{k}\right]=R_{k}=\left[\begin{array}{cc}
\sigma_{r}^{2} & 0 \\
0 & \sigma_{\phi}^{2}
\end{array}\right] .
$$

\section{B. Information exchange process}

Information exchange process between agents is typically modelled by directed or undirected graphs [20]. Suppose that there are $n$ UAVs $U=$ $\left\{U_{1}, \ldots, U_{n}\right\}$ with inconsistent information of the target. The communication pattern at time step $k$ can be expressed as a directed graph $\mathbb{G}_{k}=\left(U, \varepsilon_{k}\right)$, where $\left(U_{i}, U_{j}\right) \in \varepsilon^{k}$, if and only if there is a unidirectional information exchange link from $U_{i}$ to $U_{j}$. The adjacency matrix $G_{k}=\left[g_{k}^{i j}\right]$ of a graph $\mathbb{G}_{k}$ is defined as:

$$
g_{k}^{i j}= \begin{cases}1 & \text { if } \quad\left(U_{j}, U_{i}\right) \in \varepsilon_{k} \\ 0 & \text { if } \quad\left(U_{j}, U_{i}\right) \notin \varepsilon_{k}\end{cases}
$$

Note that $g_{k}^{i i} \triangleq 1$ for a link from each UAV to itself.

\section{Decentralised extended Information filter}

Each UAV computes local predictions based on previous and locally determined information estimates using its process model, in the form:

\section{Prediction}

$$
\begin{aligned}
\mathbf{y}_{i, k \mid k-1}^{t} & =Y_{i, k \mid k-1} F_{k} Y_{i, k-1 \mid k-1}^{-1} \mathbf{y}_{i, k-1 \mid k-1}^{t} \\
Y_{i, k \mid k-1} & =\left(F_{k} Y_{i, k-1 \mid k-1}^{-1} F_{k}^{T}+Q_{k}\right)^{-1}
\end{aligned}
$$

where $Y_{i, k}=\left(P_{i, k}\right)^{-1}$ and $\mathbf{y}_{i, k}^{t}=Y_{i, k} \mathbf{x}_{i, k}^{t}$ represent the information matrix and information state vector of the $i$-th UAV. The predictions are combined with information from local observations to compute local estimates. After exchanging these information data, information estimates for the $i$-th UAV are updated with information obtained from the other UAVs represented by $i_{j, k}$ and $I_{j, k}$ to give:

\section{Update}

$$
\begin{aligned}
& \mathbf{y}_{i, k \mid k}^{t}=\mathbf{y}_{i, k \mid k-1}^{t}+\sum_{j=1, j \neq i}^{N} g_{k}^{i j} i_{j, k} \\
& Y_{i, k \mid k}=Y_{i, k \mid k-1}+\sum_{j=1, j \neq i}^{N} g_{k}^{i j} I_{j, k}
\end{aligned}
$$

where

$$
\begin{aligned}
i_{j, k}= & H_{j, k}^{T}\left(R_{j, k}\right)^{-1} \\
& {\left[\mathbf{z}_{j, k}-h_{j}\left(\mathbf{x}_{j, k \mid k-1}^{t}\right)+H_{j, k} \mathbf{x}_{j, k \mid k-1}^{t}\right] } \\
I_{j, k}= & H_{j, k}^{T}\left(R_{j, k}\right)^{-1} H_{j, k}
\end{aligned}
$$

where subscript $j$ is associated with $j$-th UAV and the matrix $H_{j, k}$ is a Jacobian of $h_{j}$ with respect to the state $\mathbf{x}_{j, k \mid k-1}^{t}$.

\section{NUMERICAL SimUlations}

To verify the feasibility and benefits of the proposed approach, numerical simulations are performed by using realistic ground vehicle tracking scenario. The vehicle trajectory data acquired at $2 \mathrm{~Hz}$ in a S-Paramics [21] traffic model of Devizes, United Kingdom, are used to generate the GMTI measurements composed of relative range and azimuth angle with respect to a position of UAV, as shown in Fig. 6 [7]. The ground vehicle departs at the western side of Devizes and traverses a part of the town center and then turns back until the journey ends at the northwestern side of Devizes. These true GMTI measurements of UAVs were mixed with the white noise having the following 
TABLE I

PARAMETER VALUES USED IN SIMULATION

\begin{tabular}{ll}
\hline Parameters & Value \\
\hline \hline Standoff radius $r_{d}$ & $500 \mathrm{~m}$ \\
Angular separation $\theta_{d}$ & $\frac{2}{3} \pi$ \\
Desired UAV speed $V_{g}$ & $40 \mathrm{~m} / \mathrm{s}$ \\
Speed increment $\Delta V_{\max }$ & $12 \mathrm{~m} / \mathrm{s}$ \\
Time delay constant $\tau_{v}, \tau_{\omega}$ & $\frac{1}{3} \mathrm{sec}$ \\
Gain $\left[k_{v}, k_{o}, k_{I}, k_{t}, c_{1}, c_{2}\right]$ & {$[30,3,0.02,0.015,0.3,0.6]$} \\
Maximum turning rate $\omega_{\max }$ & $0.2 \mathrm{rad} / \mathrm{s}$ \\
Heading time constant $\left[\alpha, \hat{\alpha}_{0}\right]$ & {$[1.5,3.0]$} \\
Disturbance $\left[\nu, \hat{\nu}_{0}\right]$ & {$[(0.2,-0.2,0.15), 0.0]$} \\
\hline
\end{tabular}

standard deviations:

$$
\begin{array}{ll}
\text { UAV1: } & \left(\sigma_{r 1}, \sigma_{\phi 1}\right)=(10 m, 6 \text { deg }) \\
\text { UAV2: } & \left(\sigma_{r 2}, \sigma_{\phi 2}\right)=(13 m, 5 \text { deg }) \\
\text { UAV3: } & \left(\sigma_{r 3}, \sigma_{\phi 3}\right)=(15 m, 4 d e g)
\end{array}
$$

As shown in Figs. 6 7, the ground vehicle moves complicatedly with frequently changing the speed and the direction of driving. The parameters used in the simulation are shown in Table I.

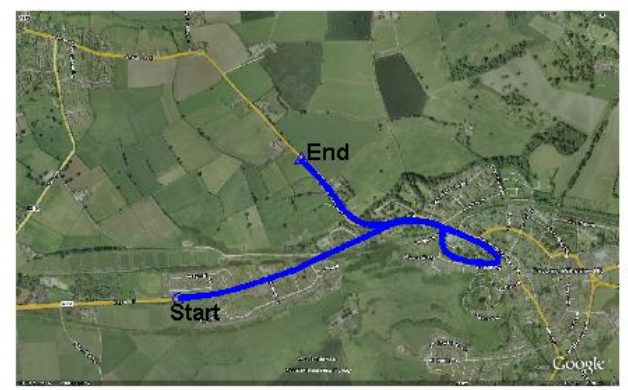

Fig. 6. The Scenario description in the civilian traffic at Devizes, Wiltshire, UK

First, ground target tracking is performed using two UAVs without coordination (i.e. no angular separation control) to investigate the performance of the TVFG using adaptive SMC. Figure 8 shows trajectories of UAVs relative to a target using with and without estimated target velocity. As can be seen in this figure, incorporating velocity information into the TVFG command greatly improves tracking guidance performance. Figure 9 displays the relative trajectories of UAVs with respect to

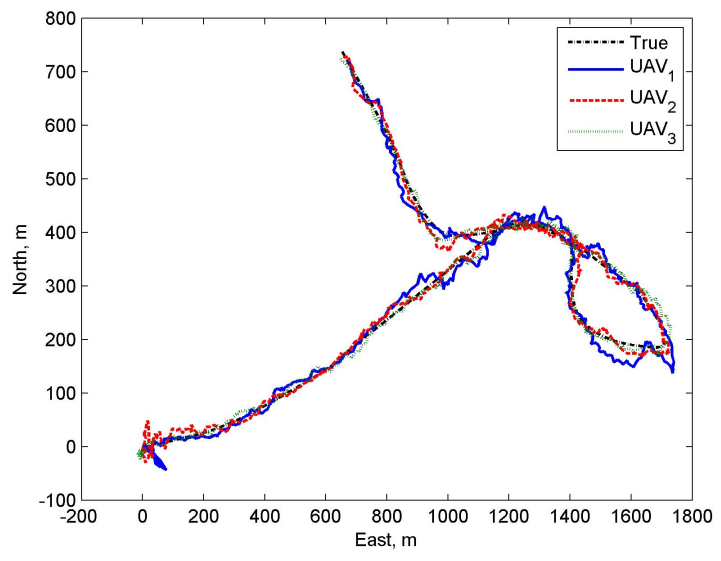

(a) Trajectory
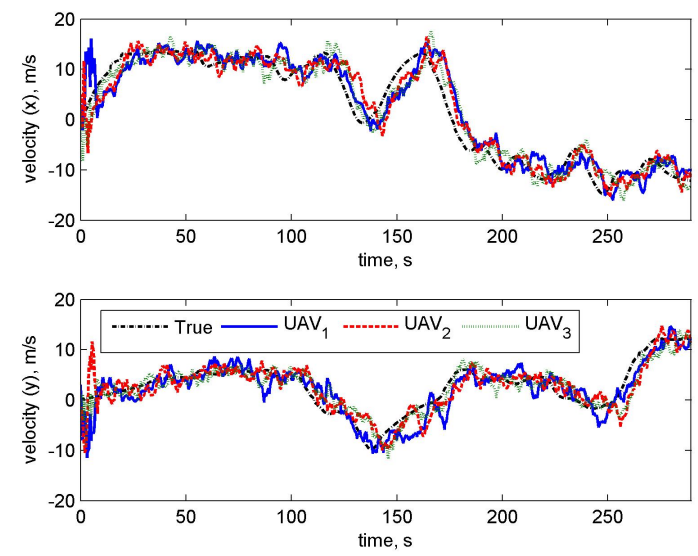

(b) Velocity

Fig. 7. Ground target estimation results using EIF

the ground vehicle, and Fig. 10 shows relative distance histories between a target and UAVs, in case that there are uncertainty and disturbances in the heading-hold loop. As can be seen in Table II, the standoff tracking performance of adaptive SMC is much better than that of normal SMC due to the estimation of unknown parameters, as shown in Fig. 11. Figure 12 shows the final absolute trajectory of a moving target and UAVs using the TVFG using adaptive SMC.

Table II shows mean errors in the decentralised estimation and the decentralised TVFG using adaptive SMC with different information/communication structures for three UAVs. In this table, 'Minimal' and 'Nonminimal' columns represent the use of a minimum communication network (i.e. only $g_{k}^{21}, g_{k}^{32}, g_{k}^{13}=1$ ) with min- 
TABLE II

PERFORMANCE COMPARISON OF GUIDANCE ALGORITHMS WITH DIFFERENT INFORMATION/COMMUNICATION STRUCTURES

\begin{tabular}{|c|c|c|c|c|c|c|}
\hline \multirow{3}{*}{ Mean error } & \multicolumn{2}{|c|}{ Single UAV (EIF) } & \multicolumn{4}{|c|}{ Multiple UAVs (DEIF) } \\
\hline & \multirow{2}{*}{ SMC } & \multirow{2}{*}{ ASMC } & \multicolumn{2}{|c|}{ Orbit radius change } & \multicolumn{2}{|c|}{ Velocity change } \\
\hline & & & Minimal & Nonminimal & Minimal & Nonminimal \\
\hline Position (m) & \multicolumn{2}{|c|}{14.3291} & 7.6990 & 4.9703 & 7.6790 & 4.7218 \\
\hline Velocity $(\mathrm{m} / \mathrm{s})$ & \multicolumn{2}{|c|}{3.1445} & 2.5216 & 2.1412 & 2.5082 & 2.0913 \\
\hline Standoff distance (m) & 26.8211 & 14.4717 & 63.3150 & 70.5503 & 15.9357 & 14.0693 \\
\hline Angular separation (deg) & - & - & 23.9987 & 16.7073 & 11.6899 & 2.7884 \\
\hline
\end{tabular}

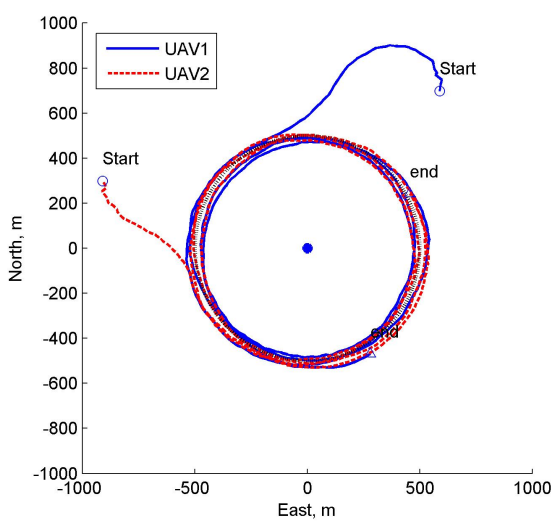

(a) Without estimated target velocity

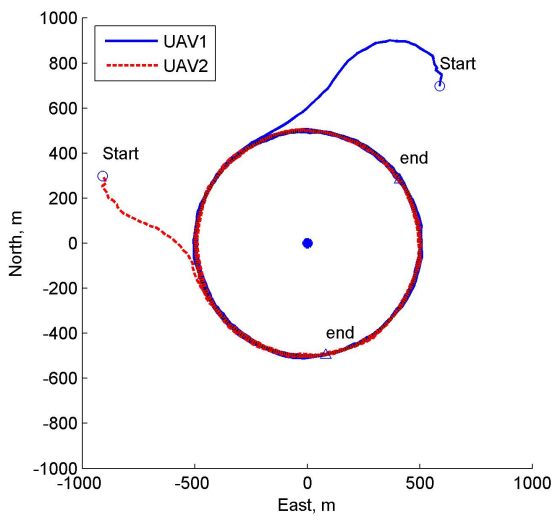

(b) With estimated target velocity

Fig. 8. Trajectories of UAVs relative to a moving target using SMC

imally persistent information architecture and a fully-connected communication link with nonmnimally persistent one, respectively. In this table and Fig. 14, the velocity change scheme with a fully-connected communication network and nonminimally information architecture shows the best performance in terms of the estimation accuracy, standoff distance, and phase keeping

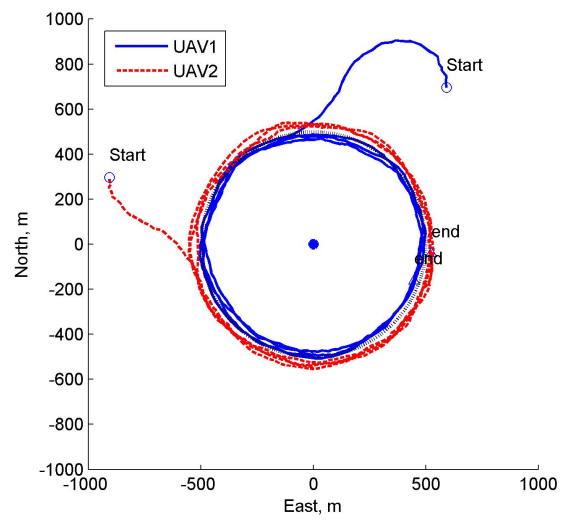

(a) $\mathrm{SMC}$

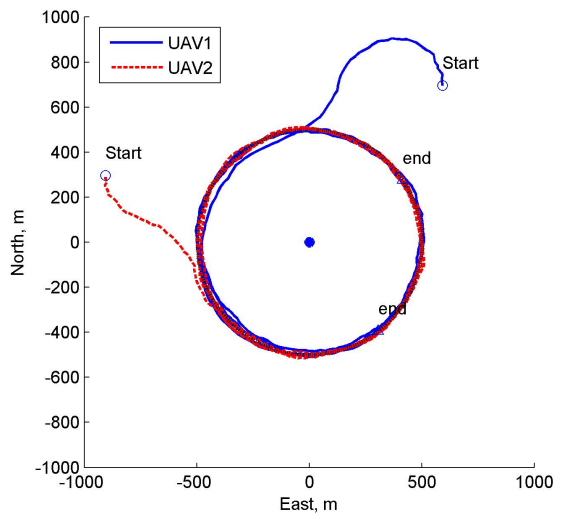

(b) Adaptive SMC

Fig. 9. Trajectories of UAVs relative to a moving target with uncertainty and disturbance in a heading-hold loop

among UAVs. However, the minimum communication/information structure requires less communication between UAVs and velocity control efforts as shown in Table II and Figs. 13 14. This can be a preferable option with its reasonable guidance performance depnding on the mission specification. Moreover, although the velocity change scheme shows much better performance than that 


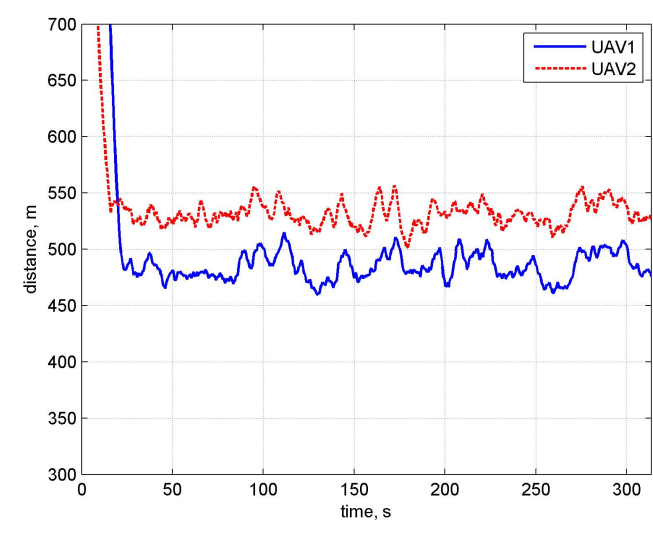

(a) SMC

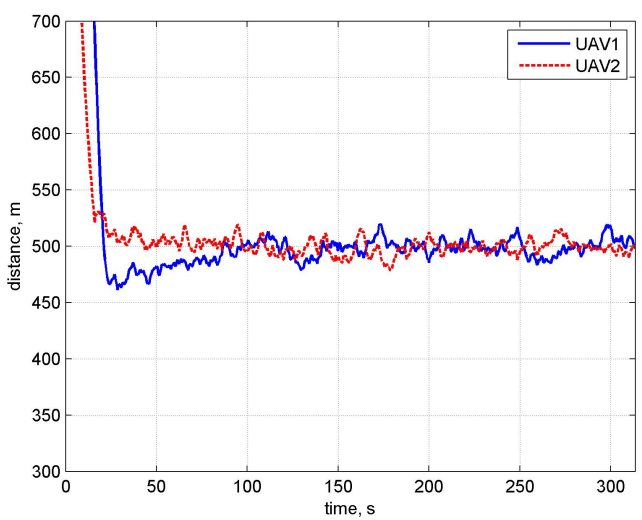

(b) Adaptive SMC

Fig. 10. Distance histories between a moving target and UAVs

of orbit radius change, in case that frequent velocity change is undesirable or unattainable, the angular separation can be achieved without velocity control but with a bounded error. Note that this is done by adjusting orbit radius appropriately at the expense of performance in standoff distance as shown in Fig. 13.

\section{CONCLUSIONS}

This paper proposed the coordinated standoff target tracking guidance algorithm using the sliding model control based on the tangent vector field. Particularly, this study used additional adaptive terms in the existing SMC approach, as well as the decentralised angular separation control using either velocity or orbit radius change by different communication/information structures. Numerical simulations for the realistic ground vehicle tracking scenario shows benefits of using adaptive terms

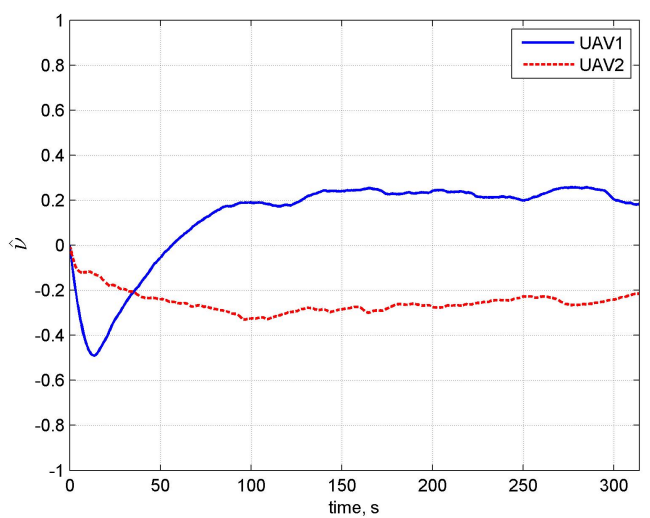

(a) Disturbance $\hat{\nu}$

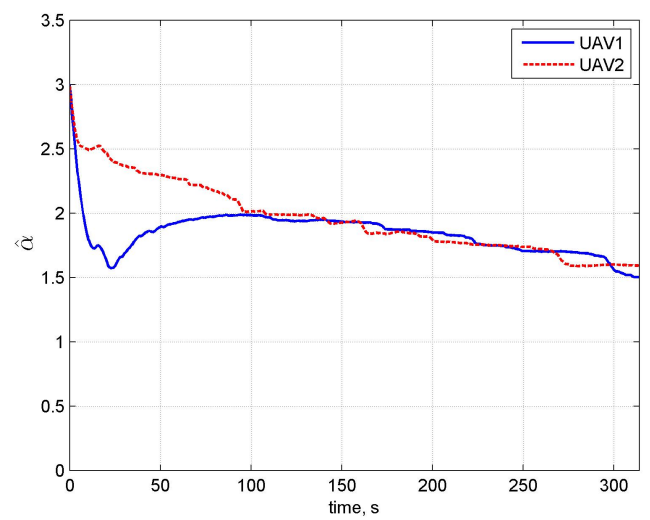

(b) Time constant $\hat{\alpha}$

Fig. 11. Estimation of unknown parameters in adaptive SMC

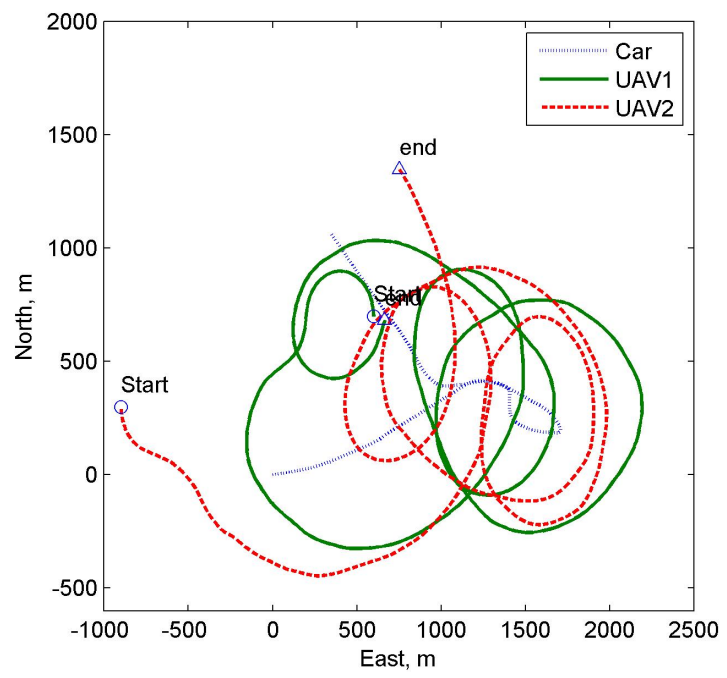

Fig. 12. Final trajectory of a moving target and UAVs 
in case that there are uncertainties in the system. Moreover, the nonminimally persistent architecture shows better performance regarding the angular separation than the minimally one. Lastly, velocity change scheme shows much better performance for the angular separation than that of radius change. However, depending on the mission requirements, the radius change could be desirable since it does not use velocity control efforts at all while achieving the angular separation within a reasonable bound.

Optimally combining proposed orbit radius and velocity change schemes for angular separation will be followed with a rigorous convergence analysis as future work, in order to obtain the better performance in terms of tracking as well as control efforts. The effect of imperfect or inconsistent information exchange between UAVs depending on communication links will also be investigated by adopting a realistic communication process.

\section{ACKNOWLEDGMENTS}

This work was partly supported by research fund of Chungnam National University.

\section{REFERENCES}

[1] M. Kim and Y. Kim. Multiple UAVs nonlinear guidance laws for stationary target observation with waypoint incidence angle constraint. International Journal of Aeronautical and Space Science, 14(1):67-74, 2013.

[2] E.W. Frew. Sensitivity of cooperative target geolocalization to orbit coordination. Journal of Guidance, Control, and Dynamics, 31(4):1028-1040, 2008.

[3] J. Kim and Y. Kim. Moving ground target tracking in dense obstacle areas using UAVs. In IFAC World Congress, COEX, South Korea, 2008.

[4] D.A. Lawrence. Lyapunov vector fields for UAV flock coordination. 2nd AIAA Unmanned Unlimited conference, workshop, and exhibit, Reston, VA, USA, 2003.

[5] E.W. Frew, D.A. Lawrence, and S. Morris. Coordinated standoff tracking of moving targets using lyapunov guidance vector fields. Journal of Guidance, Control, and Dynamics, 31(2):290-306, 2008.

[6] T.H. Summers, M.R. Akella, and M.J. Mears. Coordinated standoff tracking of moving targets: Control laws and information architectures. Journal of Guidance, Control, and Dynamics, 32(1):56-69, 2009.

[7] S. Kim, H. Oh, and A. Tsourdos. Nonlinear model predictive coordinated standoff tracking of moving ground vehicle. Journal of Guidance, Control and Dynamics, 36(2):557-566, 2013.

[8] H. Oh, S. Kim, H.S. Shin, B.A. White, A. Tsourdos, and C.A. Rabbath. Rendezvous and standoff target tracking guidance using differential geometry. Journal of Intelligent and Robotic Systems, 69:389-405, 2013.
[9] H. Oh, S. Kim, H.-S. Shin, A. Tsourdos, and B. White. Coordinated standoff tracking of groups of moving targets using multiple UAVs. In 21st Mediterranean Conference on Control and Automation, Crete, Greece, June 2013.

[10] D. Kingston and R. Beard. UAV spaly state configuration for moving targets in wind. Lecture Notes in Control and Information, 369:109-128, 2007.

[11] R. Sepulchre, D.A. Paley, and N.E. Leonard. Stabilization of planar collective motion: All-to-all communication. IEEE Transactions on Automatic Control, 52(5):811-824, 2007.

[12] D.A. Paley, N.E. Leonard, and R. Sepulchre. Stabilization of symmetric formations to motion around convex loops. Systems and Control Letters, 57:209-215, 2008.

[13] M. Quigley, M.A. Goodrich, S. Griffiths, A. Eldredge, and R.W. Beard. Target acquisition, localisation, and surveillance using a fixed-wing mini-UAV and gimbaled camera. IEEE international conference on robotics and automation, Barcelona, Spain, 2005.

[14] J.E. Marsden and M. McCracken. The Hopf Bifurcation and its applications, Vol 19 of Applied Mathematical Sciences. Springer-Verlag, 1976.

[15] M. Krstic, I. Kanellakopoulos, P.V. Kokotovic, et al. Nonlinear and adaptive control design, volume 8. John Wiley \& Sons New York, 1995.

[16] W. Koch, J. Koller, and M. Ulmke. Ground target tracking and road map extraction. ISPRS Journal of Photogrammetry and Remote Sensing, 61:197-208, 2006.

[17] A.G.O. Mutambara. Decentralized Estimation and Control for Multisensor Systems. CRC Press LLC, Boca Raton, Florida, 1998.

[18] H. Oh, S. Kim, A. Tsourdos, and B.A. White. Decentralised road-map assisted ground target tracking using a team of UAVs. In 9th IET Data Fusion \& Target Tracking, London, UK, May 2012.

[19] K. Mehrotra and P.R. Mahapatra. A jerk model for tracking highly maneuvering targets. IEEE Transactions on Aerospace and Electronic Systems, 33(4):1094-1105, 1997.

[20] W. Ren, R.W. Beard, and E.M. Atkins. A survey of consensus problems in multi-agent coordination. In American Control Conference, Portland, OR, USA, June 2005.

[21] SIAS Limited. S-paramics software. http://www.sias.com, Jan 2011. 


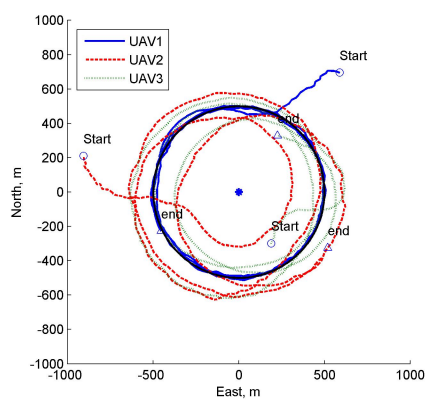

(a) Relative trajectories of UAVs

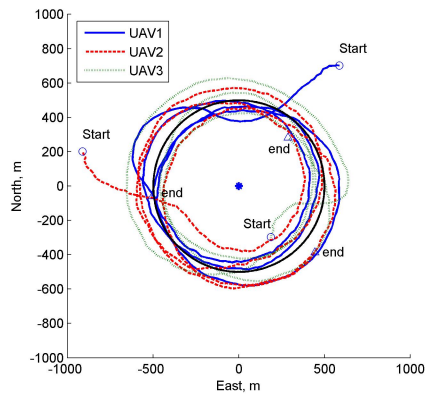

(d) Relative trajectories of UAVs

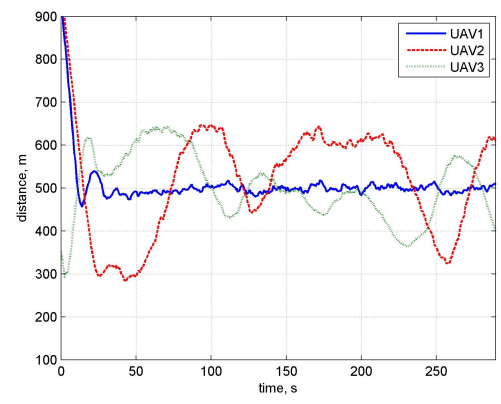

(b) Standoff distance error

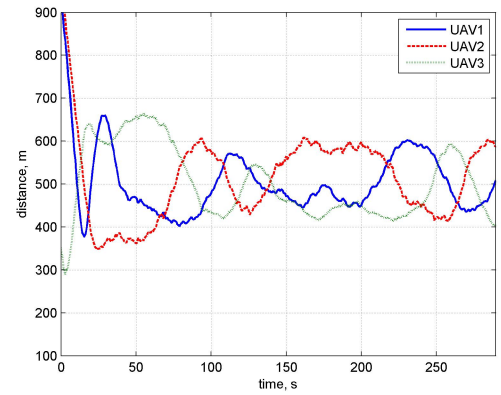

(e) Standoff distance error

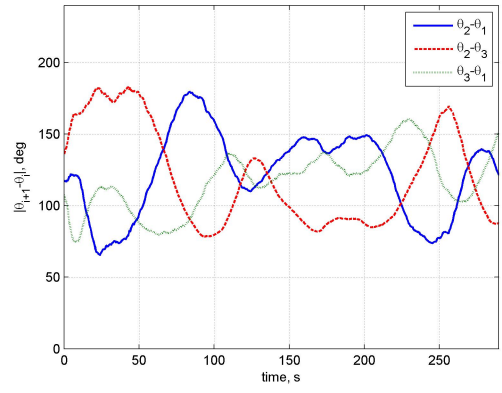

(c) Phase angle difference

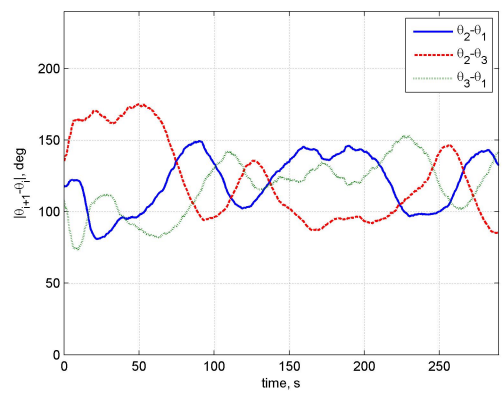

(f) Phase angle difference

Fig. 13. Standoff tracking results using orbit radius change for angular separation and DEIF: (a) (c) minimally persistent information architecture \& minimum communication link; (d) (f) nonminimally persistent \& fully-connected communication link

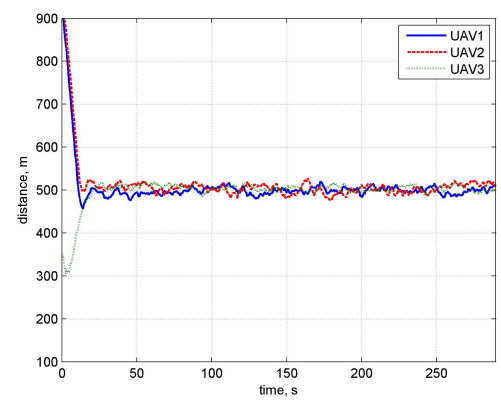

(a) Standoff distance error

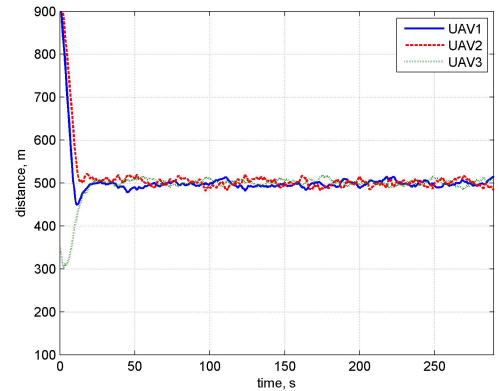

(d) Standoff distance error

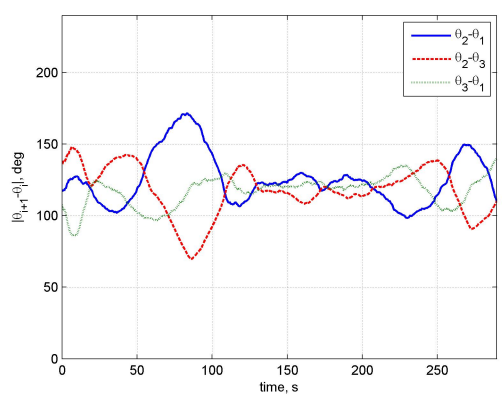

(b) Phase angle difference

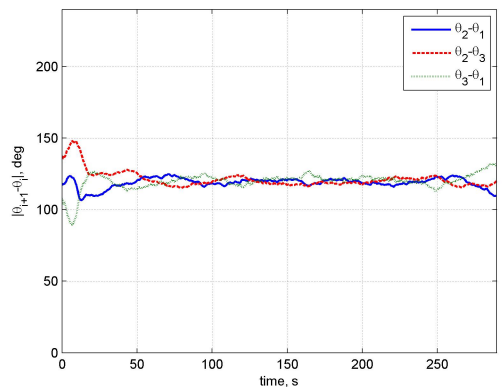

(e) Phase angle difference

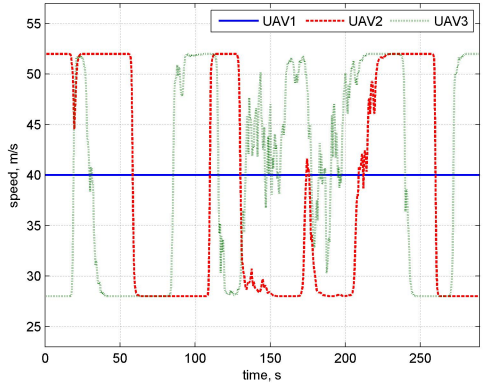

(c) Control input of UAVs: $u_{v}$

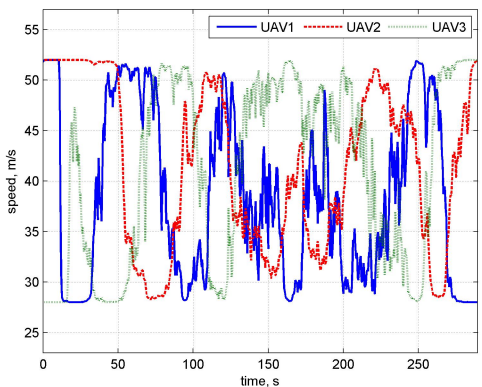

(f) Control input of UAVs: $u_{v}$

Fig. 14. Standoff tracking results using velocity change for angular separation and DEIF: (a) (c) minimally persistent information architecture \& minimum communication link; (d) (f) nonminimally persistent \& fully-connected communication link 